\title{
Experimental Realization of a Three-Dimensional Topological Insulator Phase in Ternary Chalcogenide $\mathrm{TIBiSe}_{2}$
}

\author{
K. Kuroda, ${ }^{1}$ M. Ye, ${ }^{1}$ A. Kimura, ${ }^{1, *}$ S. V. Eremeev, ${ }^{2,3}$ E. E. Krasovskii, ${ }^{4,5,6}$ E. V. Chulkov, ${ }^{4,5,7}$ Y. Ueda, ${ }^{8}$ K. Miyamoto, ${ }^{9}$ \\ T. Okuda, ${ }^{9}$ K. Shimada, ${ }^{9}$ H. Namatame, ${ }^{9}$ and M. Taniguchi ${ }^{1, \dagger}$ \\ ${ }^{1}$ Graduate School of Science, Hiroshima University, 1-3-1 Kagamiyama, Higashi-Hiroshima 739-8526, Japan \\ ${ }^{2}$ Institute of Strength Physics and Materials Science, 634021, Tomsk, Russia \\ ${ }^{3}$ Tomsk State University, 634050, Tomsk, Russia \\ ${ }^{4}$ Departamento de Física de Materiales UPV/EHU and Centro de Física de Materiales CFM and Centro Mixto CSIC-UPV/EHU, \\ 20080 San Sebastián/Donostia, Basque Country, Spain \\ ${ }^{5}$ Donostia International Physics Center (DIPC), 20018 San Sebastián/Donostia, Basque Country, Spain \\ ${ }^{6}$ IKERBASQUE, Basque Foundation for Science, 48011 Bilbao, Spain \\ ${ }^{7}$ Centro de Física de Materiales CFM-Materials Physics Center MPC, Centro Mixto CSIC-UPV/EHU, \\ Edificio Korta, Avenida de Tolosa 72, 20018 San Sebastián, Spain \\ ${ }^{8}$ Kure National College of Technology, Agaminami 2-2-11, Kure 737-8506, Japan \\ ${ }^{9}$ Hiroshima Synchrotron Radiation Center, Hiroshima University, 2-313 Kagamiyama, Higashi-Hiroshima 739-0046, Japan
}

(Received 12 July 2010; published 28 September 2010)

We report the first observation of a topological surface state on the (111) surface of the ternary chalcogenide $\mathrm{TlBiSe}_{2}$ by angle-resolved photoemission spectroscopy. By tuning the synchrotron radiation energy we reveal that it features an almost ideal Dirac cone with the Dirac point well isolated from bulk continuum states. This suggests that $\mathrm{TlBiSe}_{2}$ is a promising material for realizing quantum topological transport.

DOI: 10.1103/PhysRevLett.105.146801

PACS numbers: $73.20 .-\mathrm{r}, 79.60 .-\mathrm{i}$

Three-dimensional topological insulators, which harbor massless helical Dirac fermions in a bulk energy gap [1-4], provide fertile ground to realize new phenomena in condensed matter physics, such as a magnetic monopole arising from the topological magnetoelectric effect and Majorana fermions hosted by hybrids with superconductors $[5,6]$. All of them can hardly be achieved with trivial 2D electron gas of the semiconductor heterostructures or graphene. The topological insulator phase has been predicted to exist in a number of materials, such as $\mathrm{Bi}_{1-x} \mathrm{Sb}_{x}$, $\mathrm{Bi}_{2} \mathrm{Se}_{3}, \mathrm{Bi}_{2} \mathrm{Te}_{3}$, and $\mathrm{Sb}_{2} \mathrm{Te}_{3}$ [4]. The experimental realization of the 1st and 2nd generation of the 3D topological insulators has opened a way for applications of the quantum matter [7-12].

In particular, $\mathrm{Bi}_{2} \mathrm{Se}_{3}$ has been regarded as the most promising candidate because of its single and less-warping Dirac cone than in $\mathrm{Bi}_{2} \mathrm{Te}_{3}$. However, recent magnetotransport measurements showed that the bulk conductance dominates even in low carrier samples [13-15], which raises the question of possible scattering channels responsible for the reduced surface mobility. Band-structure calculations $[9,16]$ predict that the Dirac point (DP) of the surface state in $\mathrm{Bi}_{2} \mathrm{Se}_{3}$ is close to the bulk valence band (BVB) maximum. As a consequence, the electron scattering channel from surface states to bulk continuum states opens, and the topological transport regime collapses. Thus, it is important to extend the search for 3D topological insulators with an ideal and isolated helical Dirac cone to a wider range of materials. Recent first principles studies suggested a variety of candidates with nontrivial electronic states ranging from oxide materials, in which the electron correlation plays a role in addition to the spin-orbit coupling [17], to Heusler-type alloys with an uniaxial strain $[18,19]$.

Recently, thallium-based ternary compounds have been proposed as a new family of 3D topological insulators [20-22]. In contrast to the layered binary chalcogenides, with their inert surface due to the weak bonding between the layers, in the ternary compounds the broken bonds may give rise to trivial surface states. The theoretical studies $[21,22]$ have indeed revealed the presence of such surface states in addition to the topological ones. This calls for an angle-resolved photoemission spectroscopy (ARPES) experiment with broadly tunable photon energy, which allows us to unambiguously separate out two-dimensional electron states in this new class of ternary compounds.

The purpose of this study is twofold. The first one is to prove the existence of the Dirac surface state on the $\mathrm{TlBiSe}_{2}(111)$ surface by ARPES using tunable synchrotron radiation. The second one is to determine the energy and $\mathbf{k}$-space location of bulk continuum states with respect to the Dirac cone. Because of the strongly 3D character of the ternary compounds, a tunable photon energy is indispensable to map the 3D bulk band structure. In this Letter, we unambiguously show the presence of a single massless Dirac cone formed by the surface state in $\mathrm{TlBiSe}_{2}$. We have further revealed that $\mathrm{TlBiSe}_{2}$ features an ideal Dirac cone, which is well isolated from bulk continuum states. This suggests that the $\mathrm{TlBiSe}_{2}$ is a promising material with topological transport properties. 
(a)

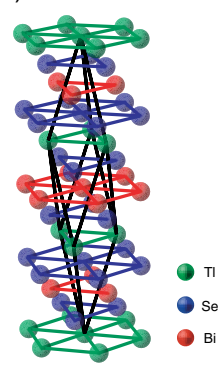

(b)

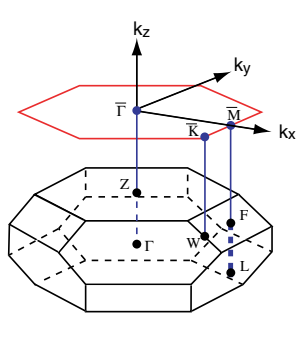

(c)

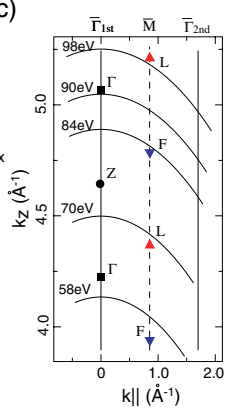

FIG. 1 (color online). (a) Rhombohedral primitive cell of $\mathrm{TlBiSe}_{2}$. The equivalent hexagonal lattice of $\mathrm{TlBiSe}_{2}$ with atomic layers stacked in the sequence -Tl-Se-Bi-Se-Tl-. (b) A schematic diagram of the bulk Brillouin zone (BZ) of $\mathrm{TlBiSe}_{2}$ and the two-dimensional $\mathrm{BZ}$ of the (111) surface. (c) $k_{z}$ plot as a function of $k_{\|}$(see text). In going from $h \nu=70$ to $98 \mathrm{eV}$, the whole Brillouin zone is scanned along the $c$ axis.

Tl-based III-V-VI 2 ternary compounds form rhombohedral crystal structure with the space group $D_{3 d}^{5}$ as shown in Fig. 1(a). This is similar to the well-known 3D topological insulator $\mathrm{Bi}_{2} \mathrm{Se}_{3}$, where five atomic layers form a quintuple layer Se-Bi-Se'-Bi-Se, and the coupling between two quintuple layers is of the van der Waals type. On the contrary, Tl-based materials have a 3D character because each $\mathrm{Tl}$ (Bi) layer is sandwiched by two Se layers with strong coupling between neighboring atomic layers. Figure $1(\mathrm{~b})$ shows the bulk and the (111) surface Brillouin zone (SBZ). Here, $\bar{\Gamma}$ and $\bar{M}$ are the time-reversal invariant wave vectors or the

surface Kramers points. The single crystalline samples of $\mathrm{TlBiSe}_{2}$ were grown by a standard procedure using Bridgman method [23]. ARPES experiment was performed with synchrotron radiation in the photon energy range of $22-98 \mathrm{eV}$ at the linear undulator beam line (BL1) of Hiroshima Synchrotron Radiation Center (HSRC). The photoemission spectra were acquired with a hemispherical photoelectron analyzer (VG-SCIENTA R4000) at $10 \mathrm{~K}$, using linearly polarized light with the electric field vector parallel ( $p$ polarization) and perpendicular ( $s$ polarization) to the plane spanned by surface normal and photoelectron propagation vector. The overall energy and angular resolutions were set at $20 \mathrm{meV}$ and $\pm 0.1^{\circ}$, respectively, which enables us to trace very steep energy dispersions. The samples were in situ cleaved under the ultrahigh vacuum below $1 \times 10^{-8} \mathrm{~Pa}$. Figure $1(\mathrm{c})$ shows the $k_{z}$ values versus $k_{\|}$at several photon energies from 58 to $98 \mathrm{eV}$ obtained with the formula $k_{z}=(1 / \hbar) \sqrt{2 m\left(E_{K} \cos ^{2} \theta+V_{0}\right)}$, where $E_{K}$ and $V_{0}$ are the photoelectron kinetic energy and inner potential. (The same value as for $\mathrm{Bi}_{2} \mathrm{Se}_{3}$ is used, $V_{0}=11.7 \mathrm{eV}$ [9].) One can thus trace the bulk electronic states along LFL direction by tuning the photon energy [Fig. 1(c)].

Figures 2(a)-2(d) show the energy dispersion curves and momentum distribution curves (MDCs) along the lines $\bar{\Gamma} \bar{M}$ and $\bar{\Gamma} \bar{K}$ of the SBZ with $s$ - and $p$-polarized light $(h \nu=58 \mathrm{eV})$. We clearly see the $X$-shaped massless energy dispersion along both high-symmetry lines. This characteristic feature remains at the same binding energy with changing the photon energy in the range $h \nu=22-98 \mathrm{eV}$,
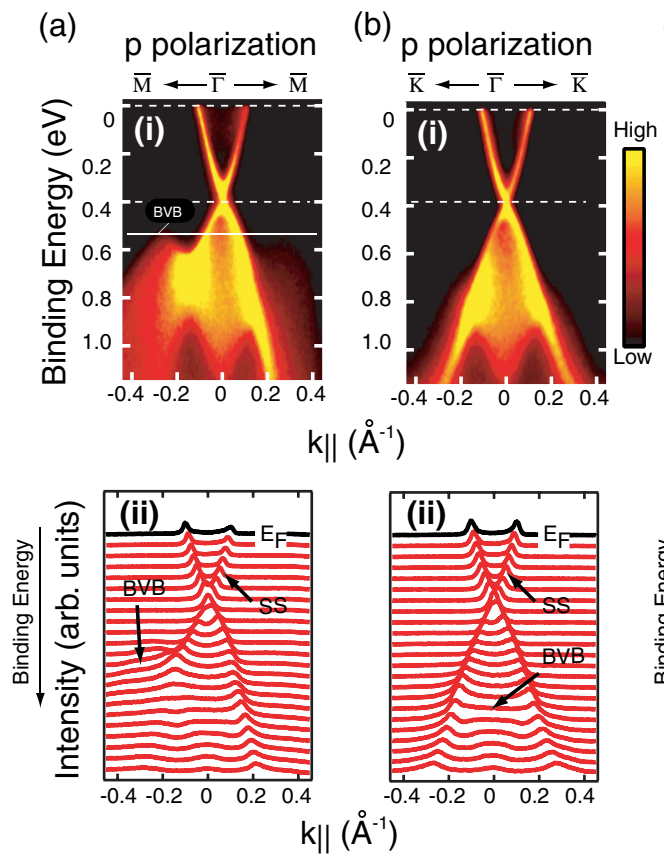

(c)

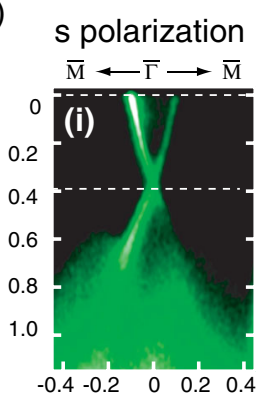

$\mathrm{k} \|\left(\AA^{-1}\right)$

(d) s polarization $\overline{\mathrm{K}} \longleftarrow \bar{\Gamma} \longrightarrow \overline{\mathrm{K}}$
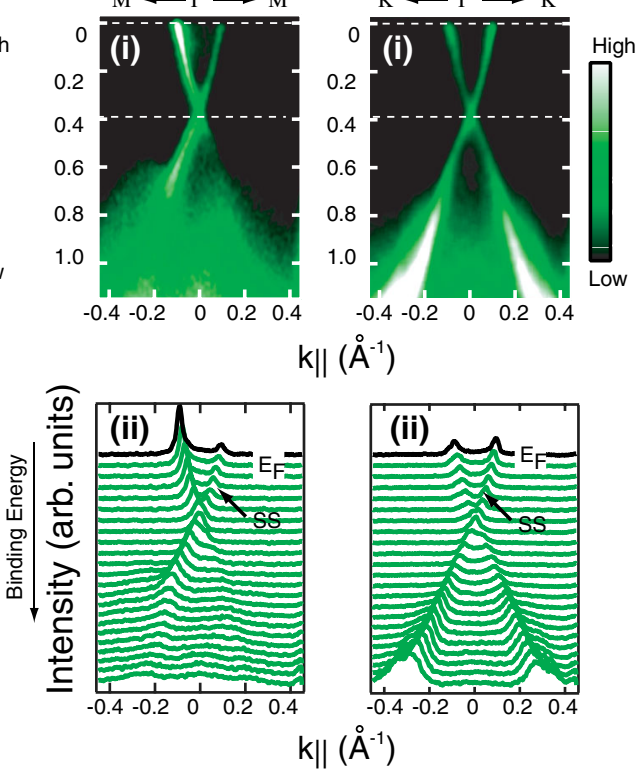

(e)

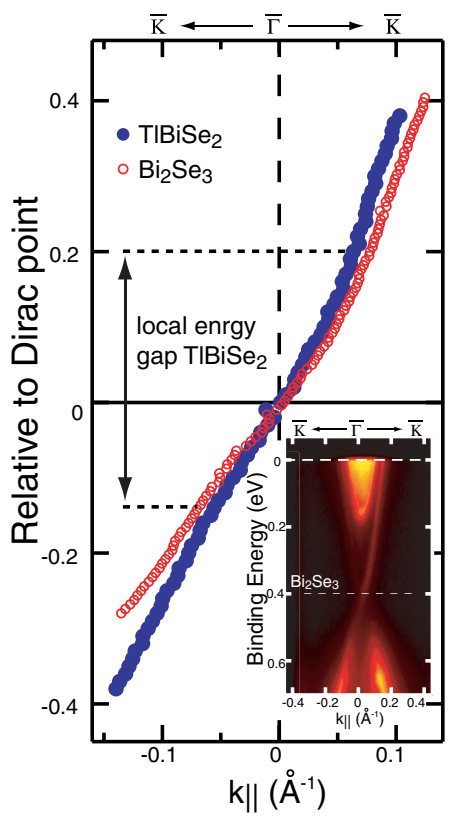

FIG. 2 (color online). ARPES on TIBiSe 2 (111): (i) $\mathbf{k}_{\|}$dispersion and (ii) momentum-space cuts measured with photon energy of $58 \mathrm{eV}$ with $p$ - and $s$-polarized light along $\bar{\Gamma} \bar{M}$ (a),(c) and $\bar{\Gamma} \bar{K}$ (b),(d) lines. (e) Intensity maxima of the surface state along $\bar{\Gamma} \bar{K}$ line together with the result for $\mathrm{Bi}_{2} \mathrm{Se}_{3}$ (ARPES energy dispersion for $\mathrm{Bi}_{2} \mathrm{Se}_{3}$ is in inset). The Dirac point is located at $E_{B}=390$ and $400 \mathrm{meV}$ for $\mathrm{TlBiSe}_{2}$ 
which confirms its two-dimensional nature. The surface state bands cross $E_{F}$ and intersect at the binding energy $E_{B}=390 \mathrm{meV}$, corresponding to the DP. The BVB energetically overlaps with the surface state below the DP and its maximum is located at $E_{B}=540 \mathrm{meV}$ away from the zone center (at $k_{\|}=0.3 \AA^{-1}$ ) as clearly seen for the $p$ polarization [Fig. 2(a)]. The BVB is strongly anisotropic: in the $\bar{\Gamma} \bar{K}$ line it lies much deeper than in the $\bar{\Gamma} \bar{M}$ line. Note that the spectral weight of the bulk conduction band (BCB) is suppressed at $h \nu=58 \mathrm{eV}$. However, the intensity of emission from the BCB strongly enhances at lower photon energies $(h \nu=22-30 \mathrm{eV}$, not shown), and the surface state signal becomes rather weak. From the spectrum at $h \nu=22 \mathrm{eV}$ we have inferred that the BCB minimum inside the cone is located at $E_{B}=200 \mathrm{meV}$. The magnitude of the Fermi wave vector is estimated from the intensity maxima of MDCs to be $0.105 \AA^{-1}$ for both $\bar{\Gamma} \bar{M}$ and $\bar{\Gamma} \bar{K}$ directions. This indicates that the ideal Dirac cone with an isotropic Fermi surface shape is expected on the $\mathrm{TlBiSe}_{2}(111)$ surface, in contrast to the heavily warped Dirac cone in $\mathrm{Bi}_{2} \mathrm{Te}_{3}$ [10,24]. When excited with $s$-polarized light, the contribution of BVB is strongly suppressed with respect to the surface state along $\bar{\Gamma} \bar{K}$ line as shown in Fig. 2(d), which enables us to evaluate the surface state character more precisely. In Fig. 2(e) we plot the energy versus $k_{\|}$of the intensity maxima for the surface state measured from the DP along $\bar{\Gamma} \bar{K}$ line and compare it with our measurements on $\mathrm{Bi}_{2} \mathrm{Se}_{3}$. The Dirac cone is seen to be not exactly linear on $\mathrm{TlBiSe}_{2}(111)$; however, it is much closer to ideally linear in $\mathrm{TlBiSe}_{2}$ than in $\mathrm{Bi}_{2} \mathrm{Se}_{3}$, especially in the local energy gap window, $150 \mathrm{meV}$ below and $200 \mathrm{meV}$ above the DP. The energy dispersions are slightly warped towards small $k_{\|}$approximately $200 \mathrm{meV}$ above the DP, where the BCB is enclosed inside the cone. The energy dispersion is steeper than in $\mathrm{Bi}_{2} \mathrm{Se}_{3}$ : the Fermi velocity is $7.1 \times 10^{5} \mathrm{~m} / \mathrm{s}$ in TIBiSe 2 and $6.6 \times 10^{5} \mathrm{~m} / \mathrm{s}$ in $\mathrm{Bi}_{2} \mathrm{Se}_{3}$. Also the velocity near the DP is larger in $\mathrm{TlBiSe}_{2}$ $\left(3.9 \times 10^{5} \mathrm{~m} / \mathrm{s}\right)$ than in $\mathrm{Bi}_{2} \mathrm{Se}_{3}\left(2.9 \times 10^{5} \mathrm{~m} / \mathrm{s}\right)$.

The knowledge of the 3D k-space location of the bulk states is important because it determines the probability of the surface-bulk electron scattering. To determine the $k_{z}$ locations we performed a detailed photon energy dependence study in a wide $k_{\|}$range. At $h \nu=84 \mathrm{eV}$, as illustrated in Fig. 1(c), the ARPES spectrum mainly reflects the electronic states at the $F$ point, which projects into the $\bar{M}$ point of the SBZ. Figure 3(a) shows the ARPES energy dispersions in the wide $k_{\|}$range along $\bar{M} \bar{\Gamma} \bar{M}$. The surface state at the $\bar{\Gamma}$ points in the 1 st $\left(\bar{\Gamma}_{1 \mathrm{st}}\right)$ and 2 nd $\left(\bar{\Gamma}_{2 \text { d }}\right)$ SBZs is confirmed to be identical. The intensity peak from the BCB moves downward as the incident photon energy increases from $74 \mathrm{eV}$ and reaches the maximal binding energy at $h \nu=84 \mathrm{eV}$ ( $F$ point), as shown in Fig. 3(a). Also, the emission from the BVB comes from near the $\bar{M}$ point at $h \nu=84 \mathrm{eV}$. In order to more clearly see the band edges at $\bar{M}$, we show in Figs. 3(b)-3(d) MDCs for three binding
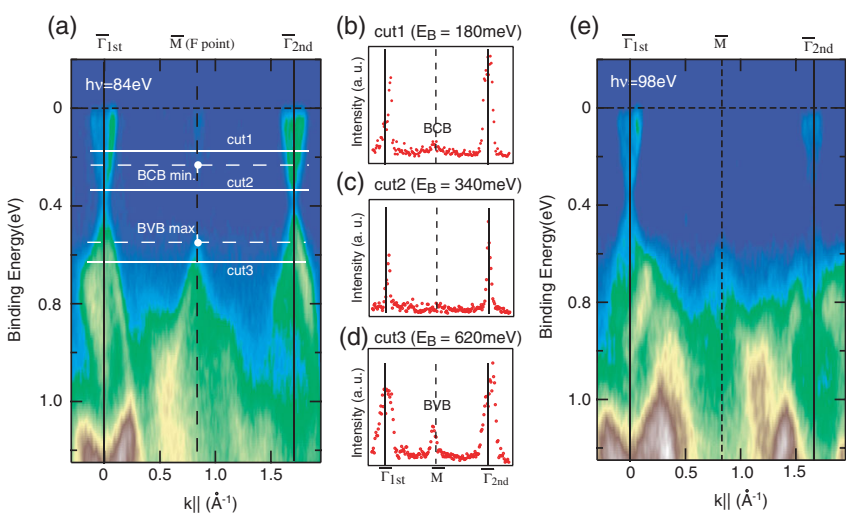

FIG. 3 (color online). (a) ARPES energy dispersions for $h \nu=84 \mathrm{eV}$ in the wide $k_{\|}$range along $\bar{M} \bar{\Gamma} \bar{M}$ together with the momentum distribution curves at the binding energies of (b) 180, (c) 340, and (d) $620 \mathrm{meV}$ (cut 1-cut 3). (e) The experimental energy dispersion for $h \nu=98 \mathrm{eV}$.

energies. From these curves we determine the conduction band minimum to be at $E_{B}=240 \mathrm{meV}$ and the valence band maximum at $E_{B}=540 \mathrm{meV}$. At $h \nu=98 \mathrm{eV}$, Fig. 3(e), which corresponds to the emission from the $L$ point, the intensity of the $\mathrm{BCB}$ vanishes.

As schematically summarized in Fig. 4(a), no states other than the Dirac cone near $\bar{\Gamma}$ reside in this energy gap region $\left(E_{B}=240-540 \mathrm{meV}\right)$. In order to see whether the experimentally derived features of the bulk band structure are consistent with theoretical models, we have performed $a b$ initio calculations using the VASP code [25]. The theoretical $\mathbf{k}_{\|}$projected bulk band structure calculated with experimental lattice parameters [23] and ideal rhombohedral atomic positions is shown in Fig. 4(b), and the result for fully optimized geometry in Fig. 4(c). The geometry optimization was performed within the generalized gradient approximation (GGA) of the density functional formalism. The band structure is seen to be extremely sensitive to small changes in geometry. Especially strong is the effect of the Se atom position: the optimization of this parameter leads to dramatic changes in the gap region (a)

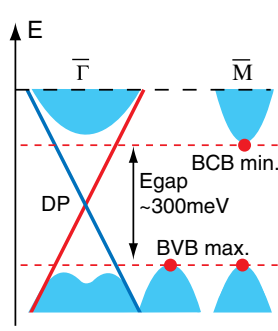

(b)

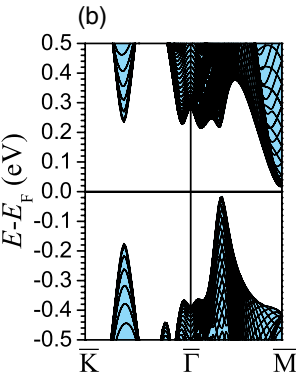

(c)

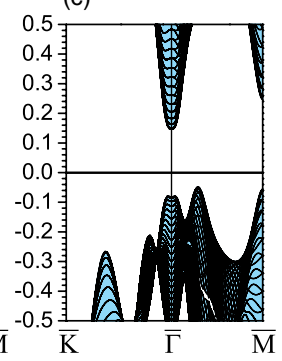

FIG. 4 (color online). (a) Schematic energy diagram of surface and bulk states of $\mathrm{TlBiSe}_{2}$ derived from the experimental results. Theoretical projected bulk band structure of $\mathrm{TlBiSe}_{2}$ obtained with ideal atomic positions (b) and with fully optimized geometry (c). Lines show constant $k_{z}$ contours. 
over the whole SBZ; in particular, it widens the indirect gap in the $\bar{\Gamma} \bar{M}$ line between $k_{\|} \approx 0.3 \AA^{-1}$ and $\bar{M}$ point and brings it close to the experimental value. At the same time, the relative locations of the BCB minima at $\bar{\Gamma}$ and at $\bar{M}$ depend on the unit cell volume (which is known to be slightly overestimated in GGA). With the fully optimized lattice the BCB minimum is at $\bar{\Gamma}$ (slightly below the minimum at $\bar{M}$ ), whereas with the experimental lattice parameters and fixed optimized fractional coordinates of the atoms the minimum is at $\bar{M}$. Thus, exact knowledge of atomic positions is absolutely necessary to reproduce the experimentally observed bulk features.

We have also performed calculations of surface electronic structure in slab geometry with relaxation of outermost atomic layers for $\mathrm{Se}, \mathrm{Tl}$, and $\mathrm{Bi}$ terminations of the surface (35-41 atomic layers). On all the surfaces we observe both the Dirac cone and the localized trivial surface states in the gap similar to those shown in Ref. [22] for $\mathrm{TlSbTe}_{2}$. They have different character and different dispersion depending on the termination, and in all cases these states are well separated from bulk bands. Our experiment, however, unambiguously rules out the presence of such states on the studied surface. The reason for that remains an open question.

In summary, our ARPES experiment of $\mathrm{TlBiSe}_{2}$ has revealed three important aspects: First, the surface state Dirac cone is confirmed to be present at the $\bar{\Gamma}$ point. Second, the Dirac cone is practically ideal, especially near the $\mathrm{DP}$, and its velocity is larger than for $\mathrm{Bi}_{2} \mathrm{Se}_{3}$. Finally, according to both experiment and theory, there are no bulk continuum states that energetically overlap with the DP. This means that the scattering channel from the topological surface state to the bulk continuum is suppressed. Our experimental results favor the realization of the topological spin-polarized transport with high mobility and long spin lifetime in $\mathrm{TlBiSe}_{2}$.

An important issue remains the apparent absence of the trivial surface states predicted by the theory: to resolve the contradiction a detailed study of the morphology and composition of the surfaces of the Tl-based ternary compounds is necessary.

We thank J. Jiang, H. Hayashi, T. Habuchi, and H. Iwasawa for their technical support in the ARPES measurement at Hiroshima Synchrotron Radiation Center (HSRC). We thank Yu. M. Koroteev for fruitful discussions. The ARPES measurement was performed with the approval of the Proposal Assessing Committee of HSRC (Proposal No. 10-A-30). This work was financially supported by the JSPS Grant-in-Aid for Scientific Research (B) 20340092. Calculations were performed on the SKIFCyberia supercomputer (Tomsk State University).

*akiok@hiroshima-u.ac.jp

${ }^{\dagger}$ Also at: Hiroshima Synchrotron Radiation Center, Hiroshima University, 2-313 Kagamiyama, HigashiHiroshima 739-0046, Japan.

[1] L. Fu, C. L. Kane, and E. J. Mele, Phys. Rev. Lett. 98, 106803 (2007).

[2] L. Fu and C. L. Kane, Phys. Rev. B 76, 045302 (2007).

[3] X. L. Qi, T. L. Hughes, and S. C. Zhang, Phys. Rev. B 78, 195424 (2008).

[4] H. Zhang, C. X. Liu, X. L. Qi, X. Dai, Z. Fang, and S. C. Zhang, Nature Phys. 5, 438 (2009).

[5] X.L Qi, R. D. Li, J.D. Zang, and S.C. Zhang, Science 323, 1184 (2009).

[6] L. Fu and C. L. Kane, Phys. Rev. Lett. 100, 096407 (2008).

[7] D. Hsieh, D. Qian, L. Wray, Y. Xia, Y. S. Hor, R. J. Cava, and M.Z. Hasan, Nature (London) 452, 970 (2008).

[8] D. Hsieh et al., Science 323, 919 (2009).

[9] Y. Xia et al., Nature Phys. 5, 398 (2009).

[10] Y. L. Chen et al., Science 325, 178 (2009).

[11] D. Hsieh et al., Nature (London) 460, 1101 (2009).

[12] A. Nishide et al., Phys. Rev. B 81, 041309 (2010).

[13] J. G. Analytis et al., Phys. Rev. B 81, 205407 (2010).

[14] K. Eto, Z. Ren, A. A. Taskin, K. Segawa, and Y. Ando, Phys. Rev. B 81, 195309 (2010).

[15] N. P. Butch et al., Phys. Rev. B 81, 241301(R) (2010).

[16] S. V. Eremeev, Yu. M. Koroteev, and E. V. Chulkov, Pis'ma Zh. Eksp. Teor. Fiz. 91, 419 (2010) [JETP Lett. 91, 387 (2010)].

[17] A. Shitade et al., Phys. Rev. Lett. 102, 256403 (2009).

[18] S. Chadov et al., Nature Mater. 9, 541 (2010).

[19] H. Lin et al., Nature Mater. 9, 546 (2010).

[20] B. Yan, C.X. Liu, H. J. Zhang, C. Y. Yam, X. L. Qi, T. Frauenheim, and S.C. Zhang, Europhys. Lett. 90, 37002 (2010).

[21] H. Lin, R. S. Markiewicz, L. A. Wray, L. Fu, M. Z. Hasan, and A. Bansil, Phys. Rev. Lett. 105, 036404 (2010).

[22] S. V. Eremeev, Yu. M. Koroteev, and E. V. Chulkov, Pis'ma Zh. Eksp. Teor. Fiz. 91, 664 (2010) [JETP Lett. 91, 594 (2010)].

[23] S. N. Toubektsis and E. K. Polychroniadis, J. Cryst. Growth 84, 316 (1987).

[24] L. Fu, Phys. Rev. Lett. 103, 266801 (2009).

[25] G. Kresse and J. Furthmüller, Phys. Rev. B 54, 11169 (1996); G. Kresse and D. Joubert, Phys. Rev. B 59, 1758 (1999). 